\title{
Estudo retrospectivo de cães com luxação patelar medial tratados cirurgicamente
}

\author{
Retrospective study of dogs with medial patellar luxation surgically treated
}

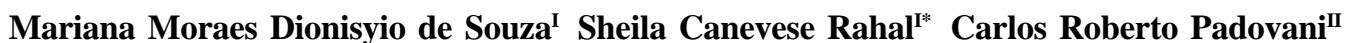 \\ Ana Carolina Mortari ${ }^{\mathrm{III}}$ Péricles Nobrega Mendes ${ }^{\mathrm{III}}$
}

\section{RESUMO}

O trabalho teve por objetivo avaliar, por meio de estudo retrospectivo, a evolução de 48 cães de porte médio a toy com luxação patelar medial de desenvolvimento submetidos a procedimentos cirúrgicos corretivos. Dos membros com grau 1 ( $n=15), 46,7 \%$ foram tratados apenas com a superposição do retináculo lateral, e 53,3\%, com a superposição do retináculo lateral e o aprofundamento do sulco troclear, não sendo detectadas complicações. Nos membros com grau $2(n=35)$, as técnicas predominantes foram aprofundamento do sulco troclear e superposição do retináculo lateral (100\% dos casos), desmotomia medial $(28,6 \%)$ e sutura fabela-patela $(2,8 \%)$, com $14,3 \%$ de recidiva da luxação. O período final de avaliação variou de três a nove meses, e sinais clínicos de claudicação ainda foram observados em $20 \%$ dos membros. Em todos os casos de grau $3(n=11)$, foram realizados aprofundamento do sulco troclear, superposição do retináculo lateral, liberação parcial do quadríceps, desmotomia medial e transposição lateral da crista da tíbia, que foi fixada com fio de Kirschner ou parafuso. Em 27,3\% dos membros, ocorreu reluxação e esses membros foram reoperados. Nesse grupo, foi detectada ausência de claudicação em 72,7\% dos membros. Nos membros com grau $4(n=10)$, além das técnicas citadas para o grau 3, foi também necessária a ostectomia femoral em quatro membros. Houve reluxação em $30 \%$ dos membros, que foram novamente operados. Embora $60 \%$ dos membros tenham apresentado uma evolução positiva, ou seja, habilidade de apoio e locomoção com os membros após o período de avaliação de 5,2 meses, em geral, os cães adotavam uma postura com os membros pélvicos em flexão, em razão da inabilidade de estender totalmente o joelho. Com base nos dados obtidos, foi possível concluir que os procedimentos cirúrgicos adotados permitiram uma melhora da locomoção; porém, com exceção do grau 1, há possibilidade de recidivas nos demais, o que indica a necessidade de realização de estudos acerca de novas condutas.

Palavras-chave: articulação, cirurgia do joelho, estabilização patelar.

\section{ABSTRACT}

The aim of this study was to evaluate retrospectively 48 medium to toy breed dogs with developmental medial patellar luxation submitted to corrective surgical procedures. In the grade 1 limbs, $46.7 \%$ were treated by lateral retinacular overlap and $53.3 \%$ by lateral retinacular overlap and deepening of the femoral groove with no complications. In the grade 2 limbs $(n=35)$ were used the techniques of deepening of the femoral groove and lateral retinacular overlap (100\% of the cases), medial desmotomy (28.6\%), fabello-patellar sutures (2.8\%) with $14.3 \%$ of reluxation. The last evaluation varied from 3 to 9 months, and clinical signs of lameness were still observed in $20 \%$ of the limbs. In all grade 3 cases $(n=11)$ were performed deepening of the femoral groove, lateral retinacular overlap, partial quadriceps released, medial desmotomy and lateral transposition of the tibial tuberosiy that was fixated with Kirschner wire or screw. Reluxation occurred in $27.3 \%$ of the limbs that were reoperated. In this group $72.7 \%$ of the limbs had absence of lameness. In the grade 4 limbs $(n=10)$ were used the same techniques of grade 3 , but it was also necessary the femoral corrective osteotomy in 4 limbs. Reluxation was observed in $30 \%$ of the limbs that were reoperated. Although $60 \%$ of the limbs showed a positive evolution of the locomotion after 5.2 months of evaluation, in general the dogs showed a posture with the hind limbs in a semi-flexed position, due to the inability to fully extend the stifle. In conclusion, the surgical procedures used allowed an improvement of the locomotion, but except to grade 1 , the patellar reluxation may occur in the other degrees suggesting the necessity of other protocols.

Key words: joint, stifle surgery, patellar stabilization.

IDepartamento de Cirurgia e Anestesiologia Veterinária, Faculdade de Medicina Veterinária e Zootecnia (FMVZ), Universidade Estadual paulista (Unesp), 18618-970, Botucatu, SP, Brasil. E-mail: sheilacr@fmvz.unesp.br. *Autor para correspondência.

IDepartamento de Bioestatística, Instituto de Biociências, Unesp Botucatu, SP, Brasil.

IIIPrograma de Pós-graduação em Medicina Veterinária, FMVZ, Unesp, Botucatu, SP, Brasil. 


\section{INTRODUÇÃO}

A luxação patelar medial é uma das afecções mais comuns em cães de raças pequenas e promove claudicação com vários graus de impotência (HULSE, 1981; HAYES et al., 1994; PIERMATTEI \& FLO, 1997; ALAM et al., 2007). A lesão é classificada de acordo com o tipo e a severidade das anormalidades em graus 1, 2, 3 e 4 (HULSE, 1981; TOMLINSON \& CONSTANTINESCU, 1994; PIERMATTEI \& FLO, 1997; L'EPLATTENIER \& MONTAVON, 2002; SCHULZ, 2007). Nos dois primeiros graus, a luxação patelar é considerada recorrente e nos dois últimos é considerada permanente (ROUSH, 1993).

O tratamento é dependente da gravidade da lesão, sendo os métodos conservativos geralmente limitados aos cães com grau 1 , sem sinais de claudicação e sem potencial para se transformar em um grau mais elevado (HULSE, 1981; ALAM et al., 2007). Os procedimentos cirúrgicos de reconstrução dos tecidos moles e ósseos (PIERMATTEI \& FLO, 1997; READ, 1999; DENNY \& BUTTERWORTH, 2000; SCHULZ, 2007) são os mais utilizados e têm por objetivo posicionar a patela adequadamente no sulco troclear, para que assim permaneça durante toda a amplitude do movimento (READ, 1999). A escolha da técnica a ser aplicada está associada à preferência e habilidade do cirurgião, porém geralmente é requerida a combinação de procedimentos conforme a anormalidade específica presente (ROUSH, 1993; TOMLINSON \& CONSTANTINESCU, 1994; ARNOCZKY \& TARVIN, 1998; SCHULZ, 2007). Em cães jovens, o ideal é que a intervenção seja empreendida o mais breve possível para evitar acentuadas anormalidades ósseas, torsionais ou angulares, secundárias a forças anormais dirigidas contra uma placa fisária aberta, em particular a do fêmur distal (HULSE, 1981; ROUSH, 1993).

A avaliação dos resultados cirúrgicos é baseada na melhora clínica, na qualidade da locomoção e no aspecto radiográfico (ROUSH, 1993). O prognóstico é dependente da severidade dos sinais clínicos, da idade do paciente no momento da intervenção e do grau de luxação patelar (ROUSH, 1993; SCHULZ, 2007). Em cães com grau 4, o sucesso pode ser limitado em virtude da extensão das anormalidades presentes (TOMLINSON \& CONSTANTINESCU, 1994). Entre as complicações pós-operatórias, são citadas a deiscência de pontos, a reluxação patelar, a migração do implante da transposição da crista tibial, o desenvolvimento de doença articular degenerativa e a incapacidade de estender o joelho (WILLAUER \& VASSEUR, 1987; ROUSH, 1993; READ, 1999; DENNY \& BUTTERWORTH, 2000; ARTHURS \& LANGLEY-
HOBBS, 2006). Essa última é frequentemente observada em cães com grau 4, quando o tratamento cirúrgico foi realizado no final do crescimento ou após 1 ano de idade (DENNY \& BUTTERWORTH, 2000).

Uma vez que o tipo de procedimento cirúrgico adotado tem relação com o sucesso e as complicações pós-operatórias, o presente trabalho teve por objetivo avaliar, por meio de estudo retrospectivo, os resultados dos métodos empregados em uma determinada população de cães.

\section{MATERIAL E MÉTODOS}

Foram avaliados retrospectivamente cães de porte médio a toy portadores de luxação medial de patela, atendidos durante um período de seis anos. Foram pesquisados, por meio de avaliação das fichas, dados referentes à identificação do animal (raça, sexo, idade e peso dos cães no momento do atendimento), ao grau de luxação patelar determinado pelo exame clínico conforme a classificação de ROUSH (1993), ao membro acometido, aos procedimentos cirúrgicos efetuados, às complicações e à evolução clínica final (presença ou ausência de claudicação), cujo tempo foi dependente do comprometimento do proprietário em comparecer aos retornos estabelecidos (Tabela 1). Os dados obtidos foram avaliados por teste de Goodman para contraste entre populações binominais (grau de luxação em relação ao sexo, grau de luxação em relação à claudicação - presença ou ausência na última avaliação clínica) e teste de análise de variância não paramétrica (teste de Krushal- Wallis), complementado com o teste de comparações múltiplas de Dunn (idade e tempo de evolução de acordo com o grau de luxação do membro), com 5\% de significância (GOODMAN, 1964; ZAR, 1999).

\section{RESULTADOS E DISCUSSÃO}

Entre as raças mais acometidas pela luxação patelar medial podem ser citados os cães Poodle toy ou miniatura, Yorkshire terrier, Chihuahua, Pomerânia, Pequinês e Boston Terrier, Cavalier King Charles Spaniel e Griffon (HULSE, 1981; ROUSH, 1993; L'EPLATTENIER \& MONTAVON, 2002). No presente estudo, de um total de 48 cães avaliados, 54,1\% pertenciam à raça Poodle $(\mathrm{n}=26) ; 18,8 \%$, à raça Pinscher $(n=9) ; 8,3 \%$ eram sem raça definida $(n=4)$; e $18,8 \%$ pertenciam a demais raças com um ou mais representantes (Tabela 1). O peso corpóreo variou de 1,3 a 37kg (média de 6,07kg), sendo 31 fêmeas (64,6\%) e 17 machos (35,4\%), porém sem diferença estatística quanto à distribuição de frequência de sexo segundo o grau (Tabela 1).

Ciência Rural, v.40, n.6, jun, 2010. 
Tabela 1 - Identificação de 48 cães com luxação patelar medial (número do caso; raça; sexo: M - macho, F - Fêmea; idade; peso corpóreo), grau de luxação (G) de acordo com o membro afetado (MPD - membro pélvico direito; MPE - membro pélvico esquerdo), recidiva da luxação após procedimento cirúrgico e período da última avaliação pós-operatória (meses) após a correção cirúrgica.

\begin{tabular}{|c|c|c|c|c|c|}
\hline Caso $\left(n^{\circ}\right)$ & Identificação (raça, sexo, idade, peso em kg) & MPD & MPE & Complicações & Última avaliação \\
\hline 1 & poodle, M, 9 anos e 1 mês, 10,2 & G2 & & & 7 \\
\hline 2 & poodle, F, 8 anos e 5 meses, 5 & & G1 & & 6 \\
\hline 3 & poodle, F, 2 anos, 1,7 & G1 & - & & 3 \\
\hline 4 & poodle, M, 3 anos, 2,6 & & G1 & & 3 \\
\hline 5 & poodle, M, 2 anos, 2,6 & G1 & & & 4 \\
\hline 6 & poodle, M, 2 anos, 6,3 & & G1 & & 3 \\
\hline 7 & poodle, F, 2 anos, 5,5 & G1 & G1 & & 3 \\
\hline 8 & poodle, F, 2 anos, 1,6 & G2 & $\mathrm{G} 2$ & & 3 \\
\hline 9 & poodle, M, 2 anos, 2,9 & & $\mathrm{G} 2$ & & 5 \\
\hline 10 & poodle, F, 8 meses, 1,7 & G2 & G2 & & 5 \\
\hline 11 & poodle, $\mathrm{M}, 3$ anos, 4,3 & & G2 & & 3 \\
\hline 12 & poodle, F, 2 anos, 2,8 & & G2 & & 3 \\
\hline 13 & poodle, $\mathrm{F}, 2$ anos, 1,7 & G2 & & & 3 \\
\hline 14 & poodle, F, 1 ano, 1,3 & G2 & & & 3 \\
\hline 15 & poodle, F, 9 anos, 3,3 & G2 & G2 & & 3 \\
\hline 16 & poodle, M, 3 anos, 4,5 & & G3 & & 3 \\
\hline 17 & poodle, F, 7 anos, 5,5 & G2 & G2 & & 3 \\
\hline 18 & poodle, F, 6 meses, 3,4 & G4 & G4 & & 10 \\
\hline 19 & poodle, M, 7 meses, 4,5 & G2 & G3 & & 3 \\
\hline 20 & poodle, F, 5 anos, 6,4 & G3 & G3 & recidiva ambos & 3 \\
\hline 21 & poodle, M, 1 ano, 4,5 & & G1 & & 3 \\
\hline 22 & poodle, M, 1 ano, 4,3 & & G1 & & 3 \\
\hline 23 & poodle, M, 2anos, 9,0 & G2 & G1 & recidiva MPD & 3 \\
\hline 24 & poodle, $\mathrm{M}, 7$ meses, 5,5 & G1 & G 2 & & 3 \\
\hline 25 & poodle, F, 2 anos, 3,8 & G3 & G3 & & 3 \\
\hline 26 & poodle, $\mathrm{F}, 2$ anos, 5,0 & G1 & & & 3 \\
\hline 27 & pinscher, F, 9 meses, 2,0 & $\mathrm{G} 2$ & & & 3 \\
\hline 28 & pinscher, M, 3 anos, 3,6 & & G2 & & 3 \\
\hline 29 & pincher, F, 2,5 anos, 2,2 & G2 & $\mathrm{G} 2$ & & 3 \\
\hline 30 & pinscher, F, 8 meses, 1,0 & G4 & G4 & recidiva ambos & 3 \\
\hline 31 & pinscher, F, 4 anos, 2,0 & G2 & $\mathrm{G} 2$ & & 3 \\
\hline 32 & pinscher, F, 1 ano, 1,8 & G4 & - & & 3 \\
\hline 33 & pinscher, F, 1 ano, 1,4 & G4 & G2 & recidiva MPD & 9 \\
\hline 34 & pinscher, F, 7 meses, 2,4 & G4 & - & & 3 \\
\hline 35 & pinscher, $\mathrm{M}, 6$ meses, 1,9 & G2 & G2 & recidiva ambos & 3 \\
\hline 36 & yorkshire, $\mathrm{M}, 1$ ano, 2,0 & G3 & G3 & & 3 \\
\hline 37 & yorkshire, F, 6 meses, 1,5 & & G1 & & 3 \\
\hline 38 & spitz, F, 4 anos e 6 meses, 2,4 & $\mathrm{G} 2$ & $\mathrm{G} 2$ & & 3 \\
\hline 39 & lhasa apso, F, 5 anos, 4,2 & G2 & G2 & recidiva ambos & 8 \\
\hline 40 & fox paulistinha, F, 6 anos e 4 meses, 9,5 & G2 & G2 & & 7 \\
\hline 41 & cocker spaniel, F, 5 anos, 9,5 & G1 & G3 & & 3 \\
\hline 42 & red heller, F, 7 meses, 9,8 & G3 & G4 & recidiva MPE & 3 \\
\hline 43 & pitt bull, F, 4 anos e 1 mês, 21 & & G2 & & 8 \\
\hline 44 & labrador, F, 3anos, 37 & G3 & & recidiva & 8 \\
\hline 45 & sem raça definida, F, 8 meses, 15 & & G2 & & 3 \\
\hline 46 & sem raça definida, F, 1 ano, 6,3 & G1 & - & & 3 \\
\hline 47 & sem raça definida, $M, 3,5$ meses, 2,5 & G4 & G4 & & 3 \\
\hline 48 & sem raça definida, $\mathrm{M}, 1$ ano, 12,4 & G1 & & & 6 \\
\hline
\end{tabular}

O processo pode ser congênito, também referido como de desenvolvimento, ou traumático, sendo o primeiro mais frequente (ROUSH, 1993; HAYES et al., 1994). Considerando que os cães foram trazidos para atendimento com idade variando entre seis meses e nove anos (média de dois anos e seis meses) e nenhum 
apresentou histórico de trauma, determinou-se que as luxações patelares eram de desenvolvimento (Tabela 1). Os cães de idade mais avançada apresentavam histórico de claudicação crônica, sendo a piora do processo provavelmente relacionada à progressão da doença articular degenerativa em virtude do mau posicionamento patelar, já que, com exceção de um cão que havia sido previamente operado de ruptura do cruzado cranial, os demais não a apresentavam. Ressalta-se que os cães com graus 1

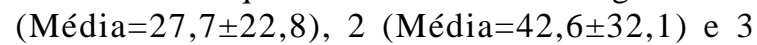
(Média=30,7 $\pm 21,3$ ) foram estatisticamente de idade mais avançada que os de grau 4 (Média=7,20 $\pm 3,1$ ). Isso se deve à maior dificuldade locomotora desses últimos, induzindo o proprietário a buscar auxílio mais cedo. Os sinais clínicos mais citados pelos proprietários foram claudicação e dificuldade locomotora, em geral, proporcional ao grau de luxação. No grau 4, os cães utilizavam predominantemente os membros torácicos para a locomoção.

A luxação foi bilateral em 23 cães (64,8\%) e unilateral em 25 (35,3\%) (Tabela 1). Segundo alguns autores, a apresentação bilateral parece ser mais prevalente que a unilateral (HAYES et al., 1994; ALAM et al., 2007). Quanto ao grau de luxação, 15 membros tinham grau 1 (21,1\%), 35 grau 2 (49,3\%), 11 grau 3 $(15,5 \%)$ e 10 grau $4(14,1 \%)$ (Tabela 1$)$. Apenas dois membros tinham sido operados previamente $(2,8 \%)$, um de ruptura do cruzado e outro de luxação patelar. Excetuando-se um cão com luxação patelar de grau 4, todos os demais com lesão bilateral tiveram os seus membros operados simultaneamente.

Dos membros com grau 1 ( $n=15), 46,7 \%$ foram tratados apenas com a superposição do retináculo lateral, e 53,3\% foram tratados com a superposição do retináculo lateral e o aprofundamento do sulco troclear. Todos os animais evoluíram sem complicações, no período de avaliação que variou de três a 10 meses (média de 3,7 meses) (Tabela 1). Nos membros com grau $2(\mathrm{n}=35)$, as técnicas predominantes foram aprofundamento do sulco troclear e superposição do retináculo lateral (100\% dos casos), desmotomia medial $(28,6 \%)$ e sutura fabela-patela (2,8\%). Destes, $14,3 \%$ apresentaram reluxação entre dois e quatro meses após a cirurgia e foram novamente operados. O período final de avaliação variou de três a nove meses (média de quatro meses), e os sinais clínicos de claudicação ainda foram observados em $20 \%$ dos membros nesse período (Tabela 1). Em todos os casos de grau $3(n=11)$, as técnicas usadas foram aprofundamento do sulco troclear, superposição do retináculo lateral, liberação parcial do quadríceps, desmotomia medial e transposição lateral da crista da tíbia, que foi fixada com fio de Kirschner ou parafuso. Um total de 27,3\% dos membros mostrou recidiva da luxação à palpação e foram submetidos à nova intervenção. No grupo, houve uma melhora da locomoção, ou seja ausência de claudicação após o tratamento cirúrgico em $72,7 \%$ dos membros, com tempo de evolução médio de 3,2 meses. Nos membros com grau $4(\mathrm{n}=10)$, além das técnicas citadas para o grau 3, foi também necessária a osteotomia femoral. Esta foi efetuada, em geral, na forma de cunha e estabilizada com emprego de placa e parafusos. Houve reluxação em 30\% dos membros, que foram novamente operados. Embora 60\% dos casos tenham apresentado uma evolução positiva, ou seja, habilidade de apoio e locomoção com os membros após o tratamento cirúrgico (média de 5,2 meses), em geral, os cães adotavam uma postura com os membros pélvicos em flexão, em razão da inabilidade de estender totalmente o joelho (Tabela 1). Observou-se uma melhor distribuição de peso entre os membros, porém esta era ainda predominante nos membros torácicos. Salientase que estatisticamente a claudicação pós-cirúrgica foi considerada relevante nos graus 3 e 4, intermediária no grau 2 e irrelevante no grau 1 , de acordo com a última avaliação clínica (Tabela 2).

Entre os métodos de reconstrução dos tecidos moles mais utilizados no tratamento da luxação patelar medial estão a superposição do retináculo ou da fáscia lata, as suturas antirrotacionais da fabela à patela ou da fabela à crista da tíbia, a desmotomia, a liberação do quadríceps e a transposição do reto femoral (PIERMATTEI \& FLO, 1997; ARNOCZKY \& TARVIN, 1998; READ, 1999; DENNY \& BUTTERWORTH, 2000; SCHULZ, 2007). Com exceção do último, indicado particularmente para cães de raças grandes que apresentam luxação medial da patela associada com acentuado arqueamento dos membros (SLOCUM \& SLOCUM, 1998; READ, 1999), todos os demais métodos foram usados no presente estudo.

Tabela 2 - Distribuição de frequência da claudicação (presente ou ausente) de acordo com o grau, na última avaliação pós-operatória de cães $(\mathrm{n}=48)$ com luxação patelar medial, totalizando 71 membros.

\begin{tabular}{llll}
\hline \multirow{2}{*}{ Grau } & \multicolumn{2}{c}{-----------Claudicação-------------- } & \\
& ausente & presente & \\
\hline 1 & $15(100,0) \mathrm{b}$ & $0(0,0) \mathrm{a}$ & 15 \\
2 & $28(80,0) \mathrm{ab}$ & $7(20,0) \mathrm{ab}$ & 35 \\
3 & $5(45,5) \mathrm{a}$ & $6(54,5) \mathrm{b}$ & 11 \\
4 & $6(60,0) \mathrm{a}$ & $4(40,0) \mathrm{b}$ & 10 \\
\hline
\end{tabular}

Valores seguidos por letras distintas na coluna diferem entre si. 
A superposição do retináculo ou da fáscia lata é uma técnica realizada para reduzir o tecido redundante e a falta de tensão rotuliana no lado oposto à luxação (READ, 1999; PIERMATTEI \& FLO, 1997). A primeira foi adotada independente do grau e se mostrou adequada para auxiliar no posicionamento da patela no sulco troclear. A desmotomia medial é necessária em todos os membros com graus 3 e 4 e em muitos que apresentam grau 2 (TOMLINSON \& CONSTANTINESCU, 1994), uma vez que alivia a contratura da cápsula articular fibrosa medial (HULSE, 1981; ROUSH, 1993), razão pela qual foi adotada no presente estudo nos graus mais acentuados. Em alguns casos grau 3 e na maioria de grau 4, o quadríceps é tão mal-alinhado que causa tensão de deslocamento na patela e precisa ser liberado muitas vezes até o nível médio femoral (PIERMATTEI \& FLO, 1997). Observouse, no entanto, que nos casos muito graves a tensão se mantinha a despeito da liberação muscular, o que contribuiu para as recidivas.

A correção das deformidades ósseas é considerada fundamental para o sucesso do tratamento da luxação patelar (READ, 1999). Entre os procedimentos de reconstrução óssea são mais comumente referidos o aprofundamento do sulco troclear, a transposição da tuberosidade tibial, a transposição da cabeça fibular, a patelectomia e as osteotomias corretivas do fêmur e da tíbia (ROUSH, 1993; DENNY \& BUTTERWORTH, 2000). No presente estudo, não foram aplicadas a transposição da cabeça fibular e a patelectomia. A primeira técnica pode ser empregada para diminuir a rotação tibial medial (ROUSH, 1993), contudo o pequeno porte dos cães utilizados dificulta a fixação da cabeça fibular. Por outro lado, a patelectomia já foi usada como tratamento paliativo em casos graves de luxação patelar (KAPLAN, 1971; READ, 1999), mas não é mais recomendada em razão dos resultados ruins em relação à função do membro(ROUSH, 1993).

O aprofundamento do sulco troclear pode ser realizado por meio da sulcoplastia troclear, condroplastia troclear ou ressecção troclear, seja esta em cunha ou bloco (ROUSH, 1993; TOMLINSON \& CONSTANTINESCU, 1994; PIERMATTEI \& FLO, 1997; SCHULZ, 2007). Este precisa ser feito de forma suficiente para acomodar pelo menos 50\% da espessura da patela (SLOCUM et al., 1982; ROUSH, 1993; TOMLINSON \& CONSTANTINESCU, 1994). Nos animais avaliados, utilizou-se em 46,5\% dos membros a ressecção troclear em cunha; em 22,5\%, a sulcoplastia troclear; e em $21,1 \%$, a condroplastia troclear.

A técnica de sulcoplastia troclear geralmente é empregada se a cartilagem articular já está danificada
(TOMLINSON \& CONSTANTINESCU, 1994). Como o osso subcondral exposto é recoberto com tecido de granulação e posteriormente fibrocartilagem, esta não possui a mesma função e durabilidade da cartilagem hialina (TOMLINSON \& CONSTANTINESCU, 1994). Por isso, no presente estudo, o aprofundamento foi efetuado com emprego de goiva e/ou lima, especialmente em casos de recidiva, presença de lesão cartilaginosa e cães de idade mais avançada.

A condroplastia troclear foi aplicada em cães com idade média de sete meses. O procedimento permite a preservação da cartilagem articular, com formação de fibrocartilagem ou tecido fibroso apenas nas lacunas da incisão, porém é limitado a pacientes jovens, tendo em vista a impossibilidade de elevação do retalho cartilaginoso no adulto (HULSE, 1981; ROUSH, 1993; PIERMATTEI \& FLO, 1997).

A técnica de resseção em cunha é uma forma de transplante autógeno osteocondral que utiliza a geometria de triângulos (SLOCUM et al., 1982). É um dos métodos preferidos de aprofundamento porque mantém o contato da patela com a cartilagem hialina normal, o que contribui para diminuir o desenvolvimento da doença articular degenerativa (ROUSH, 1993). A ressecção em bloco também preserva a cartilagem articular, com exceção que o formato é retangular (JOHNSON et al., 2001). Optou-se, no presente estudo, pela técnica em cunha, efetuada com uso de serra elétrica odontológica, que se mostrou bastante apropriada ao porte dos cães. Vale destacar que experimentalmente cães tratados pela ressecção em cunha apresentaram um retorno mais rápido à função do membro do que aqueles submetidos à sulcoplastia troclear (BOONE et al., 1983).

A transposição da tuberosidade tibial é efetuada quando a tuberosidade está anormalmente posicionada ou quando há rotação interna excessiva da tíbia (ROUSH, 1993; PIERMATTEI \& FLO, 1997; ARNOCZKY \& TARVIN, 1998). No presente estudo, esta foi usada em especial nos casos com graus 3 e 4 . O objetivo é realinhar o mecanismo do quadríceps no aspecto cranial do fêmur (ROUSH, 1993). Alguns autores sugerem que a transposição da crista tibial e/ ou trocleoplastia sejam realizadas em todos os casos de luxação patelar (ARTHURS \& LANGLEY-HOBBS, 2006). Os casos de recidiva ocorridos com o grau 2 eventualmente poderiam ter sido evitados com a inclusão do uso da transposição lateral da tuberosidade tibial.

Por sua vez, as osteotomias cuneiformes e rotacionais do fêmur ou tíbia são indicadas para os graus 4 de luxação (ROUSH, 1993; READ, 1999). A osteotomia deve ser efetuada na curvatura máxima com 
a base da cunha no lado convexo do osso (ROUSH, 1993). Em quatro membros do presente estudo, observou-se que a ostectomia em cunha fechada, na porção distal do fêmur, se fez necessária pela severa contratura dos tecidos moles que dificultavam o alinhamento do membro. Da mesma forma, ROCH E GEMMILL (2008) relataram o emprego da ostectomia em cunha fechada e fixação com placa femoral distal no tratamento de quatro cães (cinco membros) com luxação patelar recorrente. Esse tipo de procedimento provavelmente seja uma opção a ser adotada também no grau 3, já que promove um melhor alinhamento.

\section{CONCLUSÃO}

Com base nos dados obtidos, foi possível concluir que os procedimentos cirúrgicos adotados permitiram uma melhora da locomoção; porém, com exceção do grau 1, há possibilidade de recidivas nos demais, o que indica a necessidade de realização de estudos acerca de novas condutas.

\section{AGRADECIMENTOS}

Ao Conselho Nacional de Desenvolvimento Científico e Tecnológico (CNPq), pela Bolsa de Iniciação Científica PIBIC referente à Concessão da Bolsa PQ.

\section{REFERÊNCIAS}

ALAM, M.R. et al. Frequency and distribution of patellar luxation in dogs. 134 cases (2000 to 2005). Veterinary Comparative Orthopaedics and Traumatology, v.20, p.5964, 2007.

ARNOCZKY, S.P. et al. Surgical repair of patellar luxations and fractures. In: BOJRAB, M.J.; ELLISON, G.W. Current techniques in small animal surgery. 4.ed. Philadelphia: Lippincott Williams \& Wilkins, 1998. V.1, p.1237-1244.

ARTHURS, G.I.; LANGLEY-HOBBS, S.J. Complications with corrective surgery for patellar luxation in 109 dogs. Veterinary Surgery, v.35, p.559-566, 2006. Disponível em:<http:// www3.interscience.wiley.com/cgi-bin/fulltext/118621276/ PDFSTART>. Acesso em: 17 mar. 2010. doi: 10.1111/j.1532950X.2006.00189.x.

BOONE, E.G. et al. Trochlear recession wedge technique for patellar luxation: an experimental study. Journal of the American Animal Hospital Association, v.19, p.735-742, 1983.

DENNY, H.R.; BUTTERWORTH, S.J. The stifle. In: A guide to canine and feline orthopaedic surgery. United Kingdom: Blackwell Science, 2000. p.512-553.

GOODMAN, L.A. Simultaneous confidence intervals for contrasts among multinomial populations. Annals of Mathematical Statistics, v.35, n.2, p.716-725, 1964.
HAYES A.G. et al. Frequency and distribution of medial and lateral patellar luxation in dogs:124 cases (1982-1992). Journal of the American Animal Hospital Association, v.205, n.5, p.716-720, 1994.

HULSE, D.A. Pathophysiology and management of medial patellar luxation in the dog. Veterinary Medicine Small Animal Clinician, v.76, n.1, p.43-51, 1981.

JOHNSON, A.L. et al. Comparison of trochlear block recession and trochlear wedge recession for canine patellar luxation using a cadaver model. Veterinary Surgery, v.30, p.140-150, 2001. Disponível em:< http://www3.interscience.wiley.com/cgi-bin/ fulltext/119021015/PDFSTART>. Acesso em: 17 mar. 2010. doi: $10.1053 /$ jvet.2001.21391.

KAPLAN, B. Surgical palliation of bilateral congenital medial patellar luxation in a dog. Veterinary Medicine Small Animal Clinician, v.66, n. 66, p. 570-574, 1971.

L'EPLATTENIER, H.; MONTAVON, P. Patellar luxation in dogs and cats: pathogenesis and diagnosis. Compendium on Continuing Education for the Practicing Veterinarian, v.24, n.3, p.234-239, 2002.

PIERMATTEI, D.L.; FLO, G.L. The stifle joint. In: Handbook of small animal orthopedics and fracture repair. Philadelphia: Saunders, 1997. Chap.17, p.516-580.

READ, R.A. Opciones racionales de tratamiento de la luxación interna de rótula. Waltham Focus, v.9, n.4, p.25-31, 1999.

ROCH, S.P.; GEMMILL, T.J. Treatment of medial patellar luxation by femoral closing wedge ostectomy using a distal femoral plate in four dogs. Journal of Small Animal Practice, v.49, p.152-158, 2008. Disponível em:<http:// www3.interscience.wiley.com/cgi-bin/fulltext/119394003/ PDFSTART>. Acesso em: 17 mar. 2010. doi: 10.1111/j.17485827.2007.00420.x

ROUSH, J.K. Canine patellar luxation. Veterinary Clinics of North America Small Animal Practice, v.23, n.4, p.855868, 1993.

SCHULZ,. K.S. Medial patellar luxation. In: FOSSUM TW. et al. Small animal surgery. St. Louis: Mosby, 2007. p.12891297.

SLOCUM, B. et al. Wedge recession for treatment of recurrent luxation of the patella. Clinical Orthopaedics, n.164, p.4853, 1982

SLOCUM, B.; SLOCUM, T.D. Rectus femoris transposition for medial patellar luxation. In: BOJRAB, M.J. et al. Current techniques in small animal surgery. 4.ed. Philadelphia: Lippincott Williams \& Wilkins, 1998. p.1234-1237.

TOMLINSON, J.; CONSTATINESCU, G.M. Repair of medial patellar luxation. Veterinary Medicine, v.89, n.1, p.48-56, 1994.

WILLAUER, C.C.; VASSEUR, P.B. Clinical results of surgical correction of medial luxation of the patella in dogs. Veterinary Surgery, v.16, n.1, p.31-36, 1987.

ZAR, J.H. Biostatistical analysis. 4.ed. New Jersey: Prentice Hall. 1999. 663p

Ciência Rural, v.40, n.6, jun, 2010. 